\section{In Memory of Pierre Lelong}

\section{Henri Skoda, Coordinating Editor}

\section{A Tribute to Pierre Lelong}

Born in Paris on March 14, 1912, Pierre Lelong died also in Paris on October 12, 2011. He was a brilliant student and he was admitted to Ecole Normale Supérieure in 1931. He attended the lectures of Professors Arnaud Denjoy and Paul Montel, who was his thesis advisor. He defended his Thèse d'Etat in 1941 about the singularities of holomorphic functions of two variables. In 1942, he introduced the class of plurisubharmonic functions which were developed independently by Kiyoshi Oka in Japan in the early 1940s. With these functions, he built a powerful tool in complex analysis in several variables which has been clearly important in the works of many mathematicians such as K. Kodaira, H. Grauert, L. Hörmander, E. Bombieri.

Pierre Lelong taught at the Universities of Grenoble (1943-1945), Lille (1946-1954), and the University of Paris (Sorbonne and Paris 6) until 1981. From 1959 to 1961, he was a very effective advisor of the President of the French Republic Général Charles de Gaulle for scientific research and public education. Pierre Lelong was elected as a member of the French Academy of Sciences in 1985.

Henri Skoda is professor of mathematics at the University of Paris 6, Institut de Mathématiques de Jussieu. His email address is skoda@math. jussieu.fr.

Another article in honor of the memory of Pierre Lelong, with more insight into Pierre Lelong's political action, is available in the journal Normat. The reference is: Kiselman, Christer O., "Pierre Lelong 1912-2011", Nordisk matematisk tidskrift, Normat, 60 (2012), no. 2, 70-81 [2014].

DOI: http://dx.doi.org/10.1090/noti1137
He made an important contribution to the development of the French school in complex analysis and analytic geometry. One of the last great French mathematicians of the twentieth century has passed away.

\section{Jean-Pierre Demailly}

\section{Pierre Lelong: A Foundational Work in Complex Analysis and in Analytic Geometry} My first encounter with Pierre Lelong goes back to 1977 , a year during which I started to attend his "Séminaire d'Analyse" in Paris, coorganized in collaboration with Henri Skoda since 1976. During the same year, I also benefitted from a series of lectures that Pierre Lelong gave on the theory of plurisubharmonic functions and positive currents, following a Ph.D. course presented a few months earlier by Henri Skoda at Université Paris VI. These early contacts have had a strong and lasting influence on my scientific career. In fact, my later scientific investigations almost never departed from the fundamental theories initiated by Pierre Lelong during the decades 1940-1950 and 1950-1960 ([4], [5]): these theories have wonderful applications to vast subdomains of mathematics, e.g., in number theory or algebraic geometry. Even though many mathematicians throughout the world have continued exploring these directions in the following decades, most experts would certainly agree that a lot remains to be done today.

A few years later, when I defended my "Thèse de Doctorat d'État" in 1982, I had the privilege of

Jean-Pierre Demailly is professor of mathematics at the Institut Fourier, Laboratoire de Mathématiques, France. His email address is Jean-Pierre.Demai11y@ujf-grenob1e.fr. 
being invited to Pierre Lelong's private dwelling in Paris. This was the occasion for me to realize another important aspect of his past activities, namely, his deep commitment to politics, and the guidance he exerted in 1959-1961 during a major reform of higher education and research, as one of the scientific advisors of Géneral de Gaulle, then the president of the French Republic. Especially impressive were Pierre Lelong's private library and the unusual number of books and documents dealing with politics and political science. At present, the French system of higher education is faced with severe difficulties, and I cannot refrain from thinking what a benefit my country had during the 1960s, a period, of course, much more favorable economically, when the scientific policy was guided by such enlightened minds as Pierre Lelong. In fact, France enjoyed at that time a sustained scientific and technological development, as well as a very strong increase of the number of students at universities. One would like to see clearer signs today that the European governments are ready to invest in science and to give it again a prominent role in the evolution of society-and, as a consequence, to rely extensively on the expertise of the scientific community rather than on technocrats!

Even though this is probably not the most central part of Pierre Lelong's scientific work, I would like to discuss here one contribution that has in my view shed light on several important problems. This is a paper entitled "Éléments extrémaux sur le cône des courants positifs fermés" ("Extremal elements in the cone of closed positive currents"), published in the Séminaire d'Analyse in 1971/1972 [7]. The first main statement of the paper is:

Theorem 1. Let $M$ be an analytic set of complex codimension $p$, assumed to be irreducible, in a connected complex analytic manifold $X$ (countable at infinity). Then the current of integration $[M]$ is extremal in the cone of closed positive currents of bidegree $(p, p)$ in $X$.

After stating this result, Pierre Lelong observed that many other examples of closed positive currents that had then been investigated were not extremal, especially those arising from smooth convex functions or smooth plurisubharmonic exhaustion functions like $\log |z|$, and he concluded: "It is likely that Theorem 1 does not produce all extremal elements in the cone of closed positive currents; this seems to be an important unsolved question of complex geometry." I still remember a private discussion we had on this issue at Jussieu. Strongly stimulated by these observations and by a further exchange with Jean-Louis Verdier, I realized a couple of weeks later, around the end of 1981, that such a restrictive property concerning extremal elements could not hold. In fact, it would have implied via Choquet's representation theorem a formulation of the Hodge conjecture that was much too strong to be true. Therefore some of the extremal elements must be more complicated than currents of integration on analytic sets, and I found shortly afterwards an explicit example of such an extremal closed positive current of bidegree $(1,1)$ in the complex projective plane [De82]. As first noticed by Eric Bedford, further examples appear in a natural way in complex dynamics of several variables; many invariant closed positive currents produced by complex dynamical systems are actually extremal currents, although their support is in general a fractal set, and therefore is not analytic. This is, for instance, what happens for a current of the form $\lim _{k \rightarrow+\infty} d^{-k} \frac{i}{\pi} \partial \bar{\partial} \log \left|P^{k}(z)\right|$, where $P^{k}$ is the $k$ th iteration of a polynomial endomorphism of degree $d>1$ on projective space, the support of such a current being a Julia set [Sib99]. The dynamical study of "hyperbolic" endomorphisms of certain algebraic surfaces, e.g., K3 surfaces, also leads to such extremal invariant currents [2].

Another fundamental statement contained in the above-cited article [7] is the following.

Theorem 2. If $G$ is a pseudoconvex domain in $\mathbb{C}^{n}$, the positive cone generated over rational coefficients by functions of the form log $|f|$, where $f$ is holomorphic in $G$, is dense in the cone of plurisubharmonic functions on $G$.

Corollary. If, moreover, $H^{2}(G, \mathbb{R})=0$, the positive cone generated over rational coefficients by currents of integration $[D]$ on irreducible divisors of $G$ is dense in the cone of closed positive currents of type $(1,1)$ on $G$.

The original proof of Lelong rests upon a use of complex function theory on Hartogs domains of the type $|w|<e^{-\varphi(z)},(z, w) \in \mathbb{C}^{n} \times \mathbb{C}$. If $\varphi$ is plurisubharmonic and if $z$ is taken in a pseudoconvex domain $G$, it is known that the corresponding Hartogs domain in $G \times \mathbb{C}$ is again pseudoconvex; as a consequence, there exists a holomorphic function $F(z, w)$, the domain of existence of which is precisely the Hartogs domain $|w|<e^{-\varphi(z)}$. The approximation of the function $\varphi$ by logarithms of holomorphic functions $f_{j}(z)$ is 


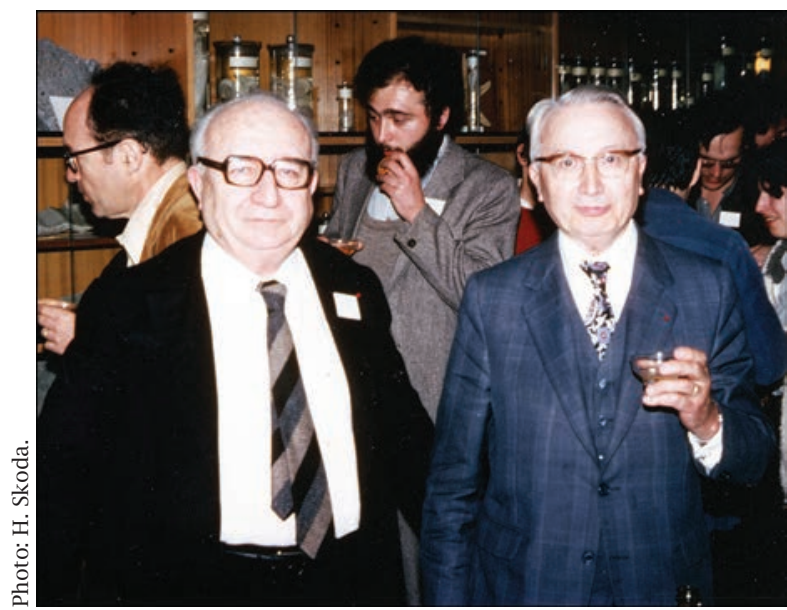

Pierre Lelong and Henri Cartan in Wimeureux, France, May 1981.

then obtained by applying Hadamard's formula to compute the radius of convergence of the power series $\sum_{k \in \mathbb{N}} a_{k}(z) w^{k}$ of $F(z, w)$. The corollary is then derived by means of the fundamental "Lelong-Poincaré" equation, stating that for every holomorphic function $F$ the current $\frac{i}{\pi} \partial \bar{\partial} \log |F|$ coincides with the current of integration $\left[Z_{F}\right]$ on the zero divisor of $F$.

These approximation results for currents are now a central ingredient of modern analytic geometry. By replacing the qualitative existence theorem of defining holomorphic functions and Hadamard's formula with deeper results such as the Ohsawa-Takegoshi $L^{2}$ extension theorem ([10]), one can obtain more precise statements in which the multiplicities of the approximating $\mathbb{Q}$-divisors converge uniformly to the "Lelong numbers" of the given closed positive $(1,1)$-current. In that way, one gets a very strong analytic tool that allows one, in particular, to prove numerous geometric resultsfor instance, Siu's theorem on the analyticity of level sets associated with Lelong numbers of closed positive currents [15]. Another consequence of such techniques in algebraic geometry is the proof of the conjecture on the invariance of plurigenera for deformations of arbitrary nonsingular projective algebraic varieties ([14], [11]); the latter result relies again on the Ohsawa-Takegoshi theorem and on a compactness argument for closed positive currents of type $(1,1)$; it comes as a surprise that no algebraic proof is known at this point in time, although the statement involves only algebraic objects!

Finally, among applications to number theory, one should mention Bombieri's theorem on algebraic values of meromorphic functions of several variables satisfying algebraic differential equations [1], [15], [9]. The proof, here again, exploits in

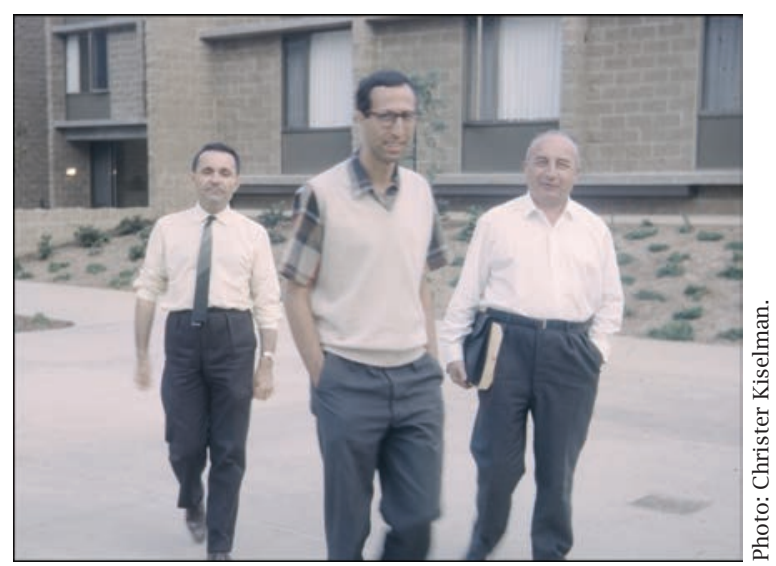

Hubert Delange, Philippe Noverraz, and Pierre Lelong in La Jolla, California, July 1966.

an essential way the compactness properties of closed positive currents of type $(1,1)$ in classes of currents of finite order, in conjunction with Hörmander's $L^{2}$ estimates for the $\bar{\partial}$ operator.

Pierre Lelong's clever use of "flexible objects"1, such as plurisubharmonic functions and positive currents, has permitted the emergence of various important techniques that have led to strong effective formulations of many results in algebraic, analytic, or arithmetic geometry, especially in areas where previously known techniques could only produce qualitative results. Pierre Lelong was perfectly aware of the philosophical dimension of the contributions he made, and he very early set up their most fundamental consequences.

\section{References}

[1] E. BOMBIERI, Algebraic values of meromorphic maps, Invent. Math. 10 (1970), 267-287; Addendum, Invent. Math. 11 (1970), 163-166.

[2] S. CANTAT, Dynamique des automorphismes des surfaces K3, Acta Math. 187 (2001), 1-57.

[3] J.-P. Demailly, Courants positifs extrêmaux et conjecture de Hodge, Invent. Math. 69 (1982), 347-374.

[4] P. LELONG, Définition des fonctions plurisousharmoniques, C. R. Acad. Sci. Paris 215 (1942), p. 398 et p. 454 .

[5] _ Intégration sur un ensemble analytique complexe, Bull. Soc. Math. France 85 (1957), 239-262.

[6] _ Fonctions plurisousharmoniques et formes différentielles positives, Dunod, Paris, Gordon \& Breach, New York (1968).

[7] _ Éléments extrémaux sur le cône des courants positifs fermés, Springer Lecture Notes in Math. 332 (1973), 112-130.

[8] _ Notice sur les titres et travaux scientifiques site de l'Académie des Sciences, http://www. academie-sciences . fr/academie/ membre/Lelong_notice_1973.pdf.

1(*) "Objets souples", according to the terminology employed by Pierre Lelong himself in his notice of scientific work sent to the Académie des Sciences [8]. 


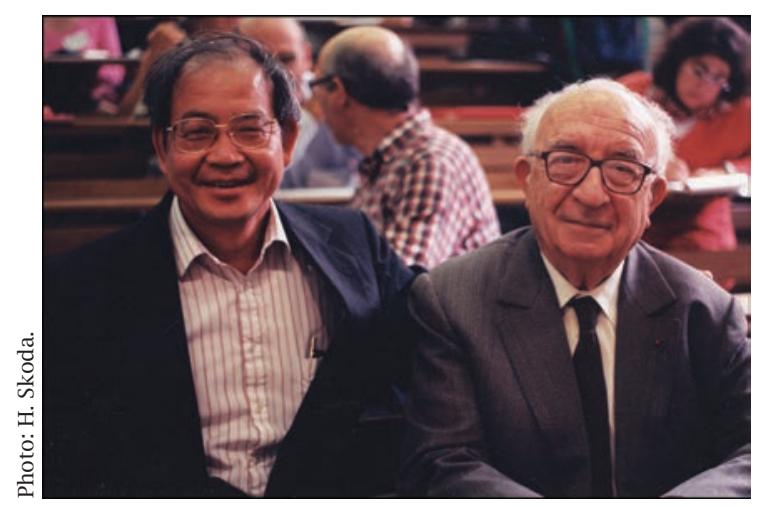

Yum Tong Siu and Pierre Lelong in Paris, September 1997.

[9] __ Sur les cycles holomorphes à coefficients positifs dans $\mathbb{C}^{n}$ et un complément au théorème de E. Bombieri, C. R. Math. Acad. Sc. Canada, vol. 1, n॰ 4 (1979), 211-213.

[10] T. OHSAWA and K. TAKEgOSHI, On the extension of $L^{2}$ holomorphic functions, Math. Zeitschrift 195 (1987) 197-204.

[11] M. PĂUN, Siu's invariance of plurigenera: A one-tower proof, J. Differential Geom. 76 (2007), 485-493.

[12] N. Sibony, Dynamique des applications rationnelles de $\mathbb{P}^{k}$, Dynamique et géométrie complexes (Lyon, 1997), ix-x, xi-xii, 97-185, Panor. Synthèses 8 Soc. Math. France, Paris (1999).

[13] Y. T. SIU, Analyticity of sets associated to Lelong numbers and the extension of closed positive currents, Invent. Math. 27 (1974), 53-156.

[14] _ Extension of twisted pluricanonical sections with plurisubharmonic weight and invariance of semipositively twisted plurigenera for manifolds not necessarily of general type, Complex Geometry (Göttingen, 2000), Springer, Berlin (2002), 223-277.

[15] H. SKODA, Estimations $L^{2}$ pour l'opérateur $\bar{\partial}$ et applications arithmétiques, Séminaire P. Lelong (Analyse), année 1975/76, Lecture Notes in Math. no. 538, Springer-Verlag, Berlin (1977), 314-323.

\section{Yum-Tong Siu}

\section{Pierre Lelong in Memoriam}

After studying his work on closed positive currents in the late 1960s, I had the opportunity of meeting Professor Pierre Lelong in person for the first time in the spring of 1972 when I visited Paris VII for one semester. He was very approachable and humorous. Though at that time I was only a young mathematician, he made me feel completely at ease in our mathematical conversations. Since then I had many occasions to discuss mathematics with him at the meetings of the Lelong-Dolbeault-Skoda Seminar and other mathematics seminars in Paris and in many conferences such as the conference

Yum-Tong Siu is professor of mathematics at Harvard University. His email address is siu@math . harvard. edu. at Poitiers in 1972, the American Mathematical Society's twenty-third summer institute at Williams College in 1975, and Lelong's seventieth birthday conference at Wimereux in 1981. I greatly enjoyed and benefitted from these conversations. Each time not only did I learn to understand better from his perspective and gain more insight into his work, but I also came away with a deepened interest and greater enthusiasm for his theory of closed positive currents. His passion for the subject was infectious.

Lelong has made many pioneering contributions in mathematics. Especially of great impact is his work on the Poincaré-Lelong equation, closed positive currents and Lelong numbers. The best way to understand this very important part of his work is to look at it from the historic perspective of constructing meromorphic functions on abstractly defined complex manifolds and see how his contributions fit in a pivotal way into the global landscape in the theory of several complex variables.

The theory of several complex variables studies complex-analytic objects such as holomorphic and meromorphic functions and maps, complexanalytic subvarieties, holomorphic vector bundles, and coherent analytic sheaves. When a complexanalytic manifold or space is projective algebraic, by definition it is embedded inside a complex projective space and there are many complexanalytic objects on it which are constructed from those on the complex projective space. When a complex-analytic manifold is abstractly defined by piecing together local holomorphic charts, the construction of complex-analytic objects on it is a problem of fundamental importance.

Historically the first breakthrough came in the form of the uniformization theorem for Riemann surfaces which states that a simply connected Riemann surface is biholomorphic to the Riemann sphere, the Gauss plane, or the open unit disk.

In the 1950s and 1960s three important methods of constructing meromorphic and holomorphic functions were introduced: Kodaira's embedding theorem, Grauert's solution of the Levi problem, and the solution of the complex Neumann problem with $L^{2}$ estimates of $\bar{\partial}$.

Kodaira's embedding theorem constructed meromorphic functions on so-called Hodge manifolds which are compact complex manifolds carrying some smooth closed positive-definite $(1,1)$-form whose cohomology class is an integer class. Kodaira proved his embedding theorem by using Hopf's blowup of a point and his vanishing theorem obtained by Bochner's technique of completion of squares applied to the quadratic polynomial whose variables are the symbols of the Laplace operator 


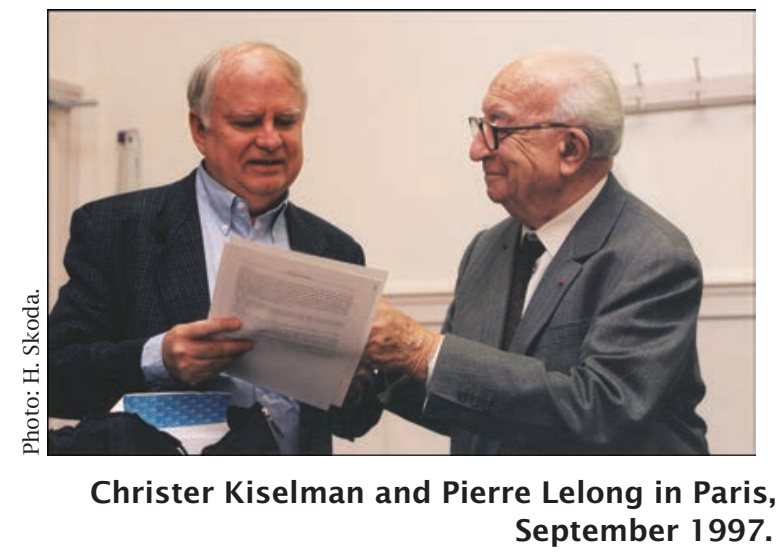

for forms with coefficients in the line bundle with Chern class represented by the given $(1,1)$-form.

Grauert's solution of the Levi problem used his bumping technique to construct holomorphic functions on complex manifolds which admit strictly plurisubharmonic exhaustion functions.

The solution of the complex Neumann problem with $L^{2}$ estimates of $\bar{\partial}$ was developed by C. B. Morrey, Kohn, Hörmander, and others. It produces holomorphic functions, with an additional $L^{2}$ growth condition, in a Levi-problem setting by applying the method of Kodaira's vanishing theorem to an open manifold, using Hörmander's modification of Friedrich's density result in the graph norm, and then using Morrey's technique of handling the boundary term by some special integration by parts involving three factors.

The significance of Kodaira's embedding, Grauert's solution of the Levi problem, and the solution of the complex Neumann problem consists in starting with soft objects like positive definite smooth $(1,1)$-forms and plurisubharmonic exhaustion functions and ending up with rigid objects like holomorphic functions.

The importance of Lelong's theory of closed positive currents is that closed positive currents introduced and studied by him are a new class of soft objects from which rigid objects like complexanalytic subvarieties can be constructed with the use of Lelong numbers.

The first step of Lelong's theory of closed positive currents is his result that integration over the regular part of a pure-dimensional complexanalytic subvariety is a closed positive current. For a complex-analytic subvariety $V$ of pure complex codimension $p$ on an open subset $G$ in $\mathbb{C}^{n}$, he proved that the $(p, p)$-current $[V]$, which is defined by integrating, over the regular part of $V$, smooth $(n-p, n-p)$-forms $\tau$ with compact support on $G$, is well defined and is a $d$-closed current. Moreover, $[V]$ is automatically positive in the sense that its value at $\tau$ is nonnegative when $\tau$ is of the form $\prod_{j=1}^{n-p} \sqrt{-1} \tau_{j} \wedge \overline{\tau_{j}}$ for some (1,0)-forms $\tau_{j}$. Lelong realized the importance of singling out the two properties of closedness and positivity for currents and developed his theory of closed positive currents.

The well-definedness and closedness of the current of integration over the regular part of a pure dimensional complex-analytic subvariety makes possible the formulation and the proof of the Poincaré-Lelong equation. In the language of currents, Cauchy's integral formula takes the form of the Poincaré formula $\frac{\sqrt{-1}}{2 \pi} \partial \bar{\partial} \log |z|^{2}=[0]$, where $z$ is the complex coordinate of $\mathbb{C}$ and [0] is the $(1,1)$-current defined by the evaluation, at the origin, of smooth functions on $\mathbb{C}$ with compact support. Lelong's result on currents defined by integration over regular parts of subvarieties enabled him to obtain the Poincaré-Lelong formula $\frac{\sqrt{-1}}{2 \pi} \partial \bar{\partial} \log |f|^{2}=[Z]$ on an open subset $G$ of $\mathbb{C}^{n}$, where $f$ is a holomorphic function on $G$ and its zeroset $Z$ is of generic multiplicity 1 . More generally, $\left(\frac{\sqrt{-1}}{2 \pi} \partial \bar{\partial} \log \sum_{j=1}^{p}\left|f_{j}\right|^{2}\right)^{p}=[V]$ on any open subset $G$ of $\mathbb{C}^{n}$, where $f_{1}, \ldots, f_{p}$ are holomorphic functions on $G$ and their common zero-set $V$ is of complex codimension $p$ and generic multiplicity 1 .

The construction of complex-analytic subvarieties from closed positive currents comes from the use of a kind of density number which Lelong introduced and which is now known as Lelong numbers. For a closed positive $(p, p)$-current $\Theta$ on an open neighborhood of a point $P$ in $\mathbb{C}^{n}$, Lelong defined the Lelong number $n(\Theta, P)$ of $\Theta$ at $P$ as the limit of the quotient of the total mass of $\Theta$ on the ball of radius $r$ in $\mathbb{C}^{n}$ by the volume of a ball of radius $r$ in $\mathbb{C}^{n-p}$. It is a number describing the density of the closed positive current $\Theta$ near the point $P$. The motivation of the Lelong number comes from the special case when $\Theta$ is the current $[V]$ defined by integration over the regular part of a complex-analytic subvariety $V$ of pure complex codimension $p$ and the Lelong number $n([V], P)$ is the multiplicity of $V$ at $P$.

The significance of Lelong numbers is that their super-level sets are closely related to complexanalytic subsets. Here a super-level set means a set consisting of all points where the value of the Lelong number is no less than a certain positive number. The first important application of the concept of Lelong numbers is Bombieri's generalization, to the higher-dimensional case by using $L^{2}$ estimates of $\bar{\partial}$, of the solution of the seventh problem of Hilbert by Gelfond and Schneider.

Later Lelong's student Skoda and other mathematicians followed in his footsteps to develop further his theory of closed positive current. Now 
a precise form of the relation between super-level sets of Lelong numbers and complex-analytic subsets is the result that for any positive number $c$ the set of points $P$ with $n(\Theta, P) \geq c$ is a complexanalytic subvariety of complex codimension $\geq p$ for any closed positive $(p, p)$-current $\Theta$.

The theory of closed positive currents is a common platform with links to the historic electrostatic potential method, Kodaira's vanishing theorem, and $L^{2}$ estimates of $\bar{\partial}$. This role of the theory of closed positive currents can be most easily seen by starting with a closer look at the historic electrostatic potential method of constructing meromorphic functions on Riemann surfaces in the proof of the uniformization theorem.

The electrostatic potential method starts with an electric charge on a Riemann surface $X$ at a point $P$ of $X$. The electrostatic potential $u$ on the Riemann surface due to the charge is constructed by minimizing the Dirichlet integral which is modified by the offset of a local electrostatic potential near $P$. The modification means subtracting from the test function an electrostatic potential on a coordinate disk centered at $P$ with vanishing boundary normal derivative and then forming the Dirichlet integral on $X$ minus the boundary of the coordinate disk. The vanishing of the boundary normal derivative of the local electrostatic potential corresponds to the Weierstrass-Erdmann corner condition in calculus of variations. The result $u$ of minimization is independent of the choice of the local coordinate disk centered at $P$ and the local offset electrostatic potential on it.

When the Riemann surface $X$ is simply connected, the normalization of the charge is chosen so that the meromorphic function whose real part is the electrostatic potential $u$ has a simple pole at $P$. This meromorphic function gives a biholomorphic map from the Riemann surface onto the entire Riemann sphere or the complement of a point or a slit in it. The local biholomorphic property of the map comes from the motivation that electric field lines outside the charge cannot intersect. The injectivity and the surjectivity onto the desired image come from the fact that domains bounded by equipotential lines and field lines must contain $P$ in their topological closures due to the minimizing of the Dirichlet integral with offset by the electrostatic potential.

There is another approach to the uniformization theorem for Riemann surfaces which uses the Riemann mapping theorem, the Schwarz reflection principle, and Koebe's distortion theorem. Unlike the approach by electrostatic potential which allows the replacement of the charge by a closed positive current in its higher-dimensional analogue, this other approach cannot be extended for use

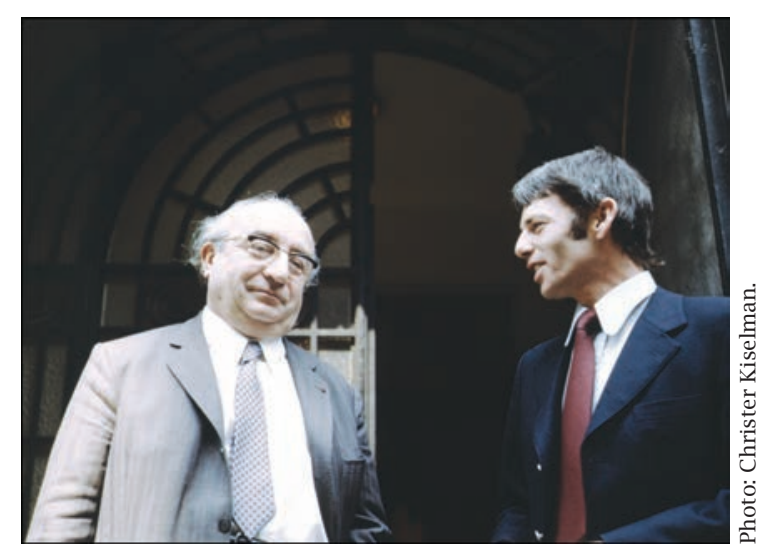

Pierre Lelong and Jean-Baptiste Poly in Paris, June 1972.

in the construction of holomorphic objects on higher-dimensional complex manifolds.

In the language of closed positive currents, for the case of the Riemann surface $X$ being the increasing union of relatively compact simply connected subdomains $X_{v}$, the potential $u$ can be obtained as the limit of $u_{v}$ which satisfies $\frac{\sqrt{-1}}{2 \pi} \partial \bar{\partial} u_{v}=-[P]$ on $X_{v}$ with the vanishing of the normal derivative of $u_{v}$ at the boundary of $X_{v}$. This is a consequence of the Weierstrass-Erdmann corner condition and the independence of $u$ on the choice of the coordinate disk at $P$. This formulation in the language of closed positive currents shares the complex Neumann condition of vanishing of boundary normal derivative with the $L^{2}$ estimates of $\bar{\partial}$ in the solution of the complex Neumann problem. One of the crucial steps of the latter is Morrey's trick of using the complex Neumann boundary condition to handle the boundary term.

The Poincaré-Lelong equation suggests that, when the open Riemann surface $X$ is replaced by a noncompact complete Kähler manifold $Y$ with $X_{v}$ replaced by a geodesic ball $Y_{v}$ of $Y$, the $(1,1)$-current $[P]$ defined by the charge at $P$ should be replaced by $[V]$ for some subvariety $V$ of pure complex codimension $p$. The electrostatic potential $u$ as the limit of $u_{v}$ is to be replaced by the limit of $v_{v}$ which satisfies the equation $\left(\frac{\sqrt{-1}}{2 \pi} \partial \bar{\partial} v_{v}\right)^{p}=[V]$ on $Y_{v}$ with the vanishing of the normal derivative of $v_{v}$ at the boundary $Y_{v}$. This partial differential equation is nonlinear for $p>1$.

For this setting Lelong's student Skoda used a related, but simpler, linear differential equation and with the use of $L^{2}$ estimates of $\bar{\partial}$, constructed, from a complex-subvariety $V$ of pure complex codimension $p$ in $\mathbb{C}^{n}$ with volume growth condition, global holomorphic functions on $\mathbb{C}^{n}$ with $L^{2}$ growth condition whose common zero-set is $V$. In the general case, with $[V]$ replaced by a a closed positive $(p, p)$-current $\Theta$, the linear differential 


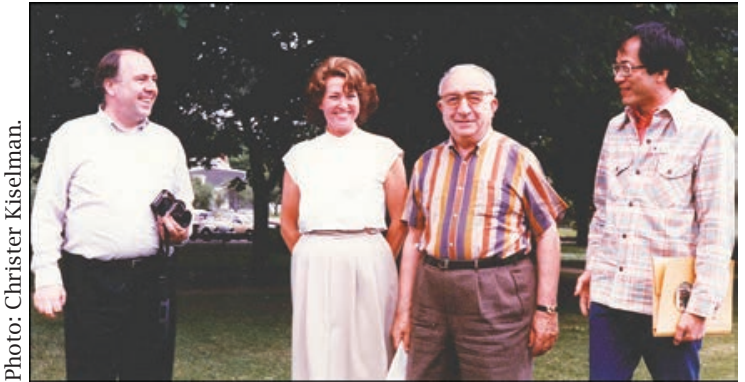

Klas Diederich, Doris Lindner, Pierre Lelong, and Hung-Hsi Wu in Wuppertal, 1986.

equation used by Skoda equates the Laplacian of the unknown function $U$ to the trace measure of $\Theta$. He then modified $U$ by a continuous function to get a plurisubharmonic function $\psi$ which he used with the method of $L^{2}$ estimates of $\bar{\partial}$ to produce his holomorphic functions. The hard analysis developed in Skoda's construction of the plurisubharmonic function $\psi$ from the closed positive current $\Theta$ provided many essential tools for the complex-analyticity of super-level sets of closed positive currents.

The method of Kodaira's vanishing theorem uses the smooth strictly positive $(1,1)$-form $\omega$ on a compact complex manifold which satisfies $\omega=\frac{\sqrt{-1}}{2 \pi} \partial \bar{\partial} \varphi$, where $e^{-\varphi}$ is the smooth metric of a holomorphic line bundle. This is analogous to the differential equation for electrostatic potential in the uniformization theorem and to the differential equation in the higher-dimensional case for a closed positive $(1,1)$-current. One difference in this analogy is that, in the setting of the uniformization theorem and the higher-dimensional situation, the manifold is noncompact and the line bundle is trivial. With the use of Lelong's theory of closed positive currents, the method of Kodaira's vanishing theorem can be generalized to the case where $\omega$ is a closed positive $(1,1)$-current which is strictly positive in the sense that it dominates some smooth positive $(1,1)$-form in the sense of currents. Now the metric $e^{-\varphi}$ is allowed to be nonsmooth so that $\varphi$ behaves like a plurisubharmonic function plus a smooth function. This generalization yielded Nadel's vanishing theorem with multiplier ideal sheaves, whose analogue in the setting of algebraic geometry is reduced to the vanishing theorem of Kawamata-Viehweg. In the 1990s the vanishing theorem for multiplier ideal sheaves was applied by Ein-Lazarsfeld, Demailly, and many others to obtain a wide range of effective results in algebraic geometry. Coupled with the use of the extension theorem of Ohsawa-Takegoshi, the vanishing theorem for multiplier ideal sheaves led also to the proof of the long-conjectured deformational invariance of plurigenera in algebraic geometry and the solution of other problems in algebraic geometry. All of these developments in the last couple of decades depend indispensably on the tools from Lelong's theory of closed positive currents.

Lelong's legacy of the theory of closed positive currents is really an amazing piece of the global jigsaw puzzle in the development of several complex variables. Without doubt it will continue to open up new vistas of research and provide ever deeper insight into the many diverse techniques in several complex variables and related fields.

\section{Henri Skoda}

\section{Pierre Lelong: A Mathematician and a Man Deeply Committed to Serving His Country}

It was not long before the events of May 1968 that I first met Pierre Lelong, at the seminar he organized jointly with François Norguet at the Institute Henri Poincaré. I was then an advanced student preparing my thesis under the supervision of André Martineau who had advised me to attend it so that I could observe "the state of the art" in the field of holomorphic functions of several complex variables. At that time, there were no individual computers nor Web. Papers were typewritten with carbon copies. Phone and television were still luxuries. So seminars were a more important place to interchange ideas and results between mathematicians than nowadays. They were fewer, and the audience of the P. Lelong and F. Norguet seminar was very impressive because of the quality and the number of its members. For instance, Henri Cartan regularly attended it. As early as October 1968, I began to study P. Lelong's works, especially those on the zeros of entire functions in $\mathbb{C}^{n}$. I discovered in them, on the one hand, the detailed study of the properties of the plurisubharmonic functions family ([Le 45]) (that is, the function is uppersemicontinuous and its restriction to every complex line is subharmonic), which included both convex functions and $\log |f|$ functions where $\mathrm{f}$ is a holomorphic function. On the other hand, as early as 1953, P. Lelong ([8]) had proved that the current of integration on a complex analytic set was well defined. In spite of the singularities of the analytic set, this current was closed. The apparent positivity of this current ([10]) (in the sense that all the measures naturally associated with that current by the complex structure were positive) led Pierre Lelong to introduce the concept of positive current. The multiplicity of the current of integration on an analytic set at a point also led P. Lelong to define, more generally, the density of a positive closed current at a point. The density was subsequently called the Lelong number of the current. In the same way as that of Laurent 
Schwartz distributions or G. de Rham currents, it gave the possibility of dealing with the analytic sets of complex geometry with analytic methods totally compatible with the algebraic method of sheaf theory and of local algebra and especially well adapted to the study of metric and quantitative properties of analytic sets. The most important part of my work is immediately connected with the concepts P. Lelong introduced. In my inaugural lecture at Colloque Européen en l'honneur de Pierre Lelong, in September 1997 ([19]), I had already widely explained the notable impact of these concepts on the development of complex analysis in several variables and on algebraic geometry on the field of complex numbers from 1940 to 1997. This in relation with Lars Hörmander ([5]), ([6]) and Enrico Bombieri ([1]) $L^{2}$ estimates for the $\bar{\partial}$ operator in 1965, then with the Ohsawa-Takegoshi ([12]) extension theorem and that of coherence from A. M. Nadel ([11]). All these results are themselves based on the notion of plurisubharmonic function as well as on a long mathematical tradition in the fields of partial differential equations and of differential geometry. In short, mathematicians could from that time on use an extremely effective machinery: by the means of a convenient integral kernel, it was possible to assign a plurisubharmonic function and then an analytic set to every closed positive current so that this analytic set was closely connected with the structure of the given current.

In this collective tribute, other mathematicians analyze this aspect of things. I would like to bring to light other aspects which are perhaps less well known. Pierre Lelong had built, as early as 1956 ([9]), the equivalent in $\mathbb{C}^{n}$ of the canonical Weierstrass product (that is, a holomorphic function $F$ of minimal growth vanishing on a given zeros set) as a plurisubharmonic potential $\log |F|$ solving in $\mathbb{C}^{n}$, in a very modern and inventive way, in the spirit of Hodge theory, the so-called (today) LelongPoincaré equation: $\frac{i}{\pi} \partial \partial \bar{l} \log |F|=[X]$ where $[X]$ is the current of integration on the hypersurface $X$. In my thesis in 1972 ([17]), I benefitted from all of these methods dealing with potential theory and $L^{2}$ estimates to extend this work of P. Lelong about hypersurfaces of $\mathbb{C}^{n}$ to any analytic set.

Then, in 1975, going back again to the solving of the same equation, Gennadi Henkin and I ([13]) were independently successful in characterizing the zeros of Nevanlinna class functions in bounded smooth strictly pseudoconvex domains of $\mathbb{C}^{n}$ by the Blaschke condition. In the same way, we more generally have solved the equation $\frac{i}{\pi} \partial \bar{\partial} V=T$ where $T$ is a closed positive current verifying the Blaschke condition.

P. Lelong's views have taken a prominent role in the following way: let $\rho$ be a smooth strictly plurisubharmonic function defining the

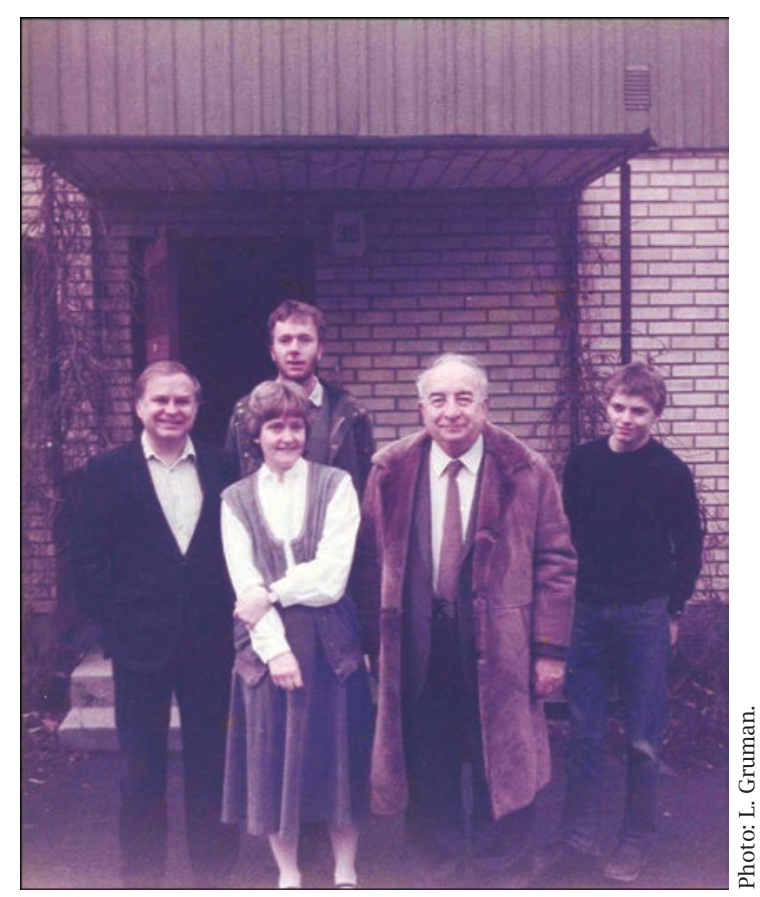

Christer Kiselman, Kiselman's wife Astrid, Bo Berndtsson, Pierre Lelong, Kiselman's son Ola, Sweden, 1981.

bounded open set $\Omega=\left\{z \in \mathbb{C}^{n} ; \rho(z)<0\right\}$. The Blaschke condition on $T$ can be written as $\int_{\Omega}-\rho(i \partial \bar{\partial} \rho)^{n-1} \wedge T<+\infty$ and Stokes's formula provides the following equality: $\int_{\Omega}-\rho(i \partial \bar{\partial} \rho)^{n-1} \wedge T=$ $\int_{\Omega}(i \partial \rho \wedge \bar{\partial} \rho) \wedge(i \partial \bar{\partial} \rho)^{n-2} \wedge T<+\infty$. This means that the complex tangential component of $T$, $(i \partial \rho \wedge \bar{\partial} \rho) \wedge T$, has finite mass in $\Omega$, hence it satisfies something stronger than the Blaschke condition. This strong condition on the behavior at the boundary of the complex tangential component of $T$ was the first compulsory and decisive step to solving the equation $\frac{i}{\pi} \partial \bar{\partial} V=T$ with the expected Nevanlinna estimate $\sup _{\epsilon>0} \int_{\rho(z)=-\epsilon} V^{+}<+\infty$. That kind of strong estimate of the complex tangential component of a closed positive current remains today as an essential argument in the numerous researches on hard analysis about zeros of functions in Hardy classes on pseudoconvex domains.

In the 1950s and 1960s, P. Lelong's ideas seemed to be very useful to control the asymptotic behavior of holomorphic objects. P. Lelong had observed that Hadamard's inequality of convexity generalized to holomorphic functions of several variables had much deeper consequences than with one variable. It implies, for instance, that the asymptotic behavior at infinity of an entire function is remarkably stable along almost all complex lines. That has been the decisive argument which was used to build ([18]) examples of holomorphic fiber spaces, with Stein $\left(\mathbb{C}^{2}\right)$ fiber and with Stein basis (an open subset 
of $\mathbb{C}$ ) but which are not Stein. It gave a negative answer to a question of Jean-Pierre Serre which has aroused the curiosity of geometers for many years. Indeed the fiber bundle is built in such a way that the action of the transition automorphisms on the fibers contradicts the consequences of the Lelong-Hadamard inequality on the growth alongside the fibers of a holomorphic function globally defined on the total space. Such a function has to be constant on the fibers and the total fiberspace is far from being Stein. This successful construction has shown that P. Lelong's methods could be more effective for strictly geometrical problems than the more traditional sheaf theoretic methods.

By chance or destiny, and probably with the help of Jean-Louis Verdier, Directeur des Études at École Normale Supérieure and Michel Hervé, Directeur Adjoint of École Normale Supérieure, one of the first who attended my lessons for advanced studies in 1976 was Jean-Pierre Demailly, a young student who was also raised on P. Lelong and L. Hörmander's methods. He immediately took up the torch by extending and making more flexible the notion of the Lelong number ([3]) and connecting it with other important problems of that time such as the Hodge conjecture ([2]) and numerical vanishing theorems. From that time forward, P. Lelong's ideas were far from seeing their end. More and more mathematicians took interest in studying his works, which had also a decisive impact on other fields, such as algebraic geometry with, for instance, the deep results of J. P. Demailly on the Fujita conjecture ([4]) and Y. T. Siu' s proof ([16]) of the invariance of plurigenera. Let us quote too the emergence of closed positive currents in the holomorphic dynamic systems with several variables, following Eric Bedford's and Nessim Sibony's works ([14]). I am grateful to Pierre Lelong for having, in an unpretentious way, laid the bases of all these mathematical developments which, I think, are far from over.

I would like now to throw light on his seminar, which became an important part of scientific life. Many French or foreign researchers were invited and benefitted from being in his audience and the broadcasting of the talks, which were published in "Seminar Acts", by Springer, between 1957 and 1986, in the Lecture Notes Series. Pierre Lelong, Pierre Dolbeault and I have shared the managing of the Complex Analysis Seminar which continues to the present day. Gennadi Henkin and Jean-Marie Trépreau have taken part in the seminar management. From October 2006, with the arrival of Olivier Bicquard and Tien Cong Dinh, it has become the Geometry and Complex Analysis Seminar and has quite turned to differential geometry, dealing, however, with an important part of complex analysis. From time to time, Henri Cartan and Laurent Schwartz attended the seminar, and more regularly Paul Malliavin and Michel Hervé. In organizing it, we could talk not only about mathematics, research, and university, but also about the part involving administration and state in the research field.

I immediately saw in P. Lelong's speech and individual characteristics the mark of the humanist tradition, reinforced by his classical studies in secondary school, which gives more importance to man than to ideology and technology. It was in that way P. Lelong undertook to serve the state. He trained for public service for a long time, by attending, in the 1930s, the Institut Politique de Paris, in addition to his mathematical activities. In the 1960s, he became a scientific consultant of the president of the French Republic, Général Charles de Gaulle, and so he gave a contribution to the effort for the expansion of universities and planning for research. He especially contributed to the expansion of computer science in France with the establishment of INRIA. I think his influence has been highly beneficial and we owe him much still today. He also tried, during the 1980s, to protect the Institute Henri Poincaré (IHP). For, because of a legal vacuum, mathematicians could have been excluded from this institute. He did his best to obtain clear statutes for IHP, acting in the best interest of mathematicians and theoretical physicists.

I wish to pay tribute to the memory of Pierre Lelong. One of the best mathematicians of the twentieth century has left us, whose influence will go on for a long time, but who was also an academic deeply wedded to humanistic values and to funding the values of the French Republic.

\section{References}

[1] E. BOMBIERI, Algebraic values of meromorphic maps, Invent. Math. 10 (1970), 267-287; and addendum, Invent. Math. 11 (1970), 163-166.

[2] J-P. Demailly, Courants positifs extrêmaux et conjecture de Hodge, Invent. Math. 69 (1982), 457-511.

[3] _ Nombres de Lelong généralisés, théorèmes d'intégralité et d'analyticité, Acta Math. 159 (1987), 153-169.

[4] _ A numerical criterion for very ample line bundles, J. Differential Geom. 37 (1993), 323-374.

[5] L. HÖRMANDER, $L^{2}$ Estimates and existence theorem for the $\bar{\partial}$ operator, Acta Math. 113 (1965), 89-152.

[6] __ An Introduction to Complex Analysis in Several Variables, 1966, 2nd edition, North Holland Math. Libr., Vol 7, Amsterdam, London, 1990.

[7] P. Lelong, Les fonctions plurisousharmoniques, Ann. Sci. Ec. Norm. Sup. Paris 62 (1945), 301-338.

[8] _ Intégration sur un ensemble analytique complexe, Bull. Soc. Math. France 85 (1957), 239-262.

[9] __ Fonctions entières ( $n$ variables) et fonctions plurisousharmoniques d'ordre fini dans $\mathbb{C}^{n}$, Jour. d'Analyse (Jerusalem) 12 (1964), 365-406. 
[10] _ Plurisubharmonic Functions and Positive Differential Forms, Gordon and Breach, New York, and Dunod, Paris, 1969.

[11] A. M. NADEL, Multiplier ideal sheaves and KählerEinstein metrics of positive scalar curvature, Proc. Nat. Acad. Sci. U.S.A. 86 (1989), 7299-7300; and Annals of Math. 132 (1990), 549-596.

[12] T. OHSAWA, On the extension of $L^{2}$ holomorphic functions, II, Publ. RIMS, Kyoto Univ. 24 (1988), 265-275.

[13] W. RuDIN, Function Theory in the Unit-Ball of $\mathbb{C}^{n}$, 1980, Springer Verlag, New York.

[14] N. SibONY, Dynamique des applications rationnelles de $\mathbb{P}^{k}$, Dynamique et Géométrie complexe (Lyon 1997), ix-x, xi-xii, 97-185, Panor. et Synthèses 8, Soc. Math. France, Paris (1999).

[15] Y. T. SIU, Analyticity of sets associated to Lelong numbers and the extension of closed positive currents, Invent. Math. 27 (1974), 53-156.

[16] _ Invariance of Plurigenera, Invent. Math. 134 (1998), 662-673.

[17] H. SKODA, Sous-ensembles analytiques d'ordre fini ou infini dans $\mathbb{C}^{n}$, Bull. Soc. Math. Fr. 100 (1972), 353-408.

[18] _ Fibrés holomorphes à base et à fibre de Stein, Inventiones Math. 43 (1977), 97-107.

[19] _ _ Présence de l'oeuvre de P. Lelong dans les grands thèmes de recherche d'aujourd'hui, Complex Analysis and Geometry, International Conference in Honor of Pierre Lelong, (1997), Progress in Mathematics, vol. 188, Basel, Boston, Berlin. Birkhäuser (2000), $1-30$.
Quecriterizisse

- COUNT

LIKE AN

EGYPTIAN

tomentersent

DAVID REIMER

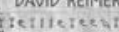

Q areniziesa

Aleasio

ilaminges

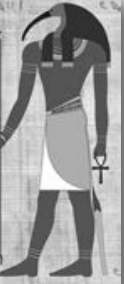

Count Like an

Egyptian

A Hands-on Introduction to Ancient Mathematics

David Reimer

"This book is by far the best presentation of Egyptian math I have read. In an age of overpopularized and sensationalized science reporting, Reimer's crisp prose and concise exposition earned my unqualified admiration. Count Like an Egyptian is destined to become a classic."

-Eli Maor, author of $e$ : The Story of a Number

Cloth \$29.95 978-0-691-16012-2
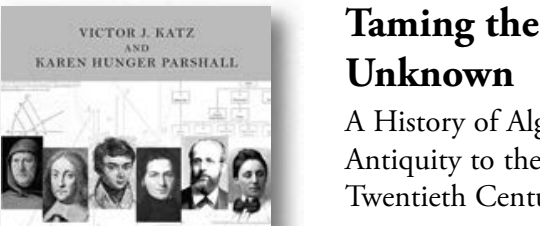

A History of Algebra from Antiquity to the Early Twentieth Century Victor J. Katz \&

TAMING THE

UNKNOWN

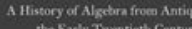

the Barly Thentieth Cenaury

"Taming the Unknown is well written and informative, and will satisfy any reader with an interest in the history of algebra. Striking just the right balance between general overview and technical detail, this book is a pleasure to read." - Joseph W. Dauben, City University of New York, Graduate Center

Cloth \$49.50 978-0-691-14905-9

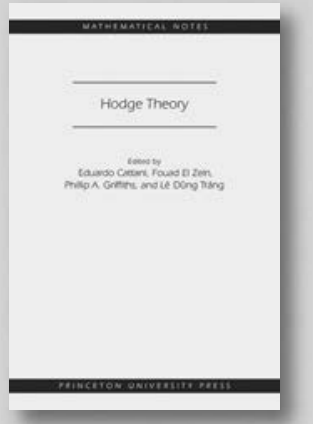

\section{Hodge Theory} Edited by Eduardo Cattani, Fouad El Zein, Phillip A. Griffiths \& Lê Düng Tráng

This book provides a comprehensive and up-to-date introduction to Hodge theory-one of the central and most vibrant areas of contemporary mathematics-from leading specialists on the subject. The topics range from the basic topology of algebraic varieties to the study of variations of mixed Hodge structure and the Hodge theory of maps.

Paper $\$ 90.00$ 978-0-691-16134-1 